\title{
LE STATUT JURIDIQUE EN DROIT INTERNE DES ESPĖCES ANIMALES LIÉES AUX MILIEUX AQUATIQUES CONTINENTAUX MÉTROPOLITAINS.
}

\author{
V. BENTATA \\ Ministère de l'Environnement - Direction de la Nature et des Paysages, \\ 20 avenue de Ségur, 75302 Paris 07 SP, France.
}

\section{RÉSUMÉ}

La loi "Barnier" ( $n^{\circ}$ 95-101 du 2 février 1995), relative au renforcement de la protection de l'Environnement, a introduit dans le code rural deux articles (L.211-3 et L.211-4) qui traitent de l'introduction d'espèces dans le milieu naturel. Ces nouveaux articles prennent place dans un cadre préexistant constitué par l'ensemble des régimes juridiques dont relèvent, en France, de très nombreuses espèces de la faune sauvage. Les introductions d'espèces étant un phénomène ancien, il est intéressant d'examiner la structure de ce dispositif et d'en profiter pour repérer à quelles places il avait, jusque-là, intégré des espèces qui, pour avoir été introduites en France avant la loi "Barnier ", n'en étaient pas pour autant dénuées de statut juridique.

A grands traits, on peut proposer de reconnaître quatre grandes catégories de listes d'espèces de faune sauvage : la faune protégée, les gibiers, les animaux de pêche, et enfin les espèces relevant de classifications agricoles. La faune protégée comprend non seulement ce qu'il est convenu d'appeler les "espèces protégées" (celles qui figurent sur des listes prises au titre des articles L.211-1 et L.211-2 du code rural), mais aussi d'autres espèces figurant sur des listes prises au titre de l'article L.212-1 de ce code (en particulier, celles des annexes de la convention de Washington et du règlement (CEE) $N^{\circ} 3626 / 82$ du conseil du 3 décembre 1982 , modifié, relatif à son application dans la Communauté). Le gibier comprend notamment trois ensembles, qui s'emboîtent comme des poupées russes : le gibier au sens de la jurisprudence (le plus vaste), les espèces dont la chasse est autorisée, et enfin celles d'entre elles qui sont susceptibles d'être classées "nuisibles" par les préfets. A noter que ces deux derniers ensembles sont définis par la voie réglementaire. Les animaux de pêche, au sens large (qui regroupent des crustacés et des grenouilles avec les poissons et les agnathes), font l'objet de listes diverses : espèces représentées dans les eaux douces soumises à la législation sur la pêche ; espèces susceptibles de provoquer des déséquilibres biologiques ; espèces citées au $3^{\circ}$ de l'article L.232-10 du code rural ; espèces non représentées, mais figurant sur la liste ministérielle de celles dont l'introduction peut être autorisée en vertu d'un arrêté préfectoral pris au titre de l'article R. ${ }^{* 232-8 ~ d u ~ c o d e ~ r u r a l ~ ; ~ a u t r e s ~ e s p e ̀ c e s ~ n o n ~ r e p r e ́ s e n t e ́ e s, ~ m a i s ~}$ d'introduction autorisée en vertu d'une décision ministérielle prise au titre de l'article R."232-9 du code rural. Les classifications agricoles interviennent aussi, dans la mesure où l'article 342 du code rural prévoit l'établissement d'une liste d' " organismes nuisibles " aux végétaux ou aux produits végétaux, différente de celle - évoquée plus haut - qui concerne exclusivement certaines espèces de gibiers.

Il est également utile d'examiner quelques catégories remarquables d'espèces qui ne figurent pas sur ces listes. Ainsi : les espèces visées par des listes négatives ; certaines espèces omises sur des listes positives ; différents cas particuliers de mammifères ; certaines espèces d'animaux d'ornement ou d'aquariophilie... 
On conclut par l'examen des possibilités d'appartenance d'une même espèce à différentes catégories juridiques, et par l'examen du poids relatif que prennent respectivement espèces indigènes et espèces introduites dans ces catégories.

Mots-clés : statuts juridiques, espèces animales, introductions. milieux aquatiques continentaux, France métropolitaine.

\title{
LEGAL STATUS OF ANIMAL SPECIES LINKED TO CONTINENTAL FRENCH (GUYANE EXCLUDED) AQUATIC ENVIRONMENTS IN DOMESTIC LAW.
}

\begin{abstract}
The "Barnier" Law (95-101 of 2 February 1995), the general objective of which was the reinforcement of the protection of the environment, inserted two new sections (L.211-3 and L.211-4) dealing with the introduction of species into the natural environment in the French Rural Code. These sections are new parts of the comprehensive set of existing legal regulations that concern a number of wild species in France. Species introduction being an ancient practice, it seemed interesting to review the criteria used for classifying the various legal statutes and determining those applied to certain species that had been introduced into France before the "Barnier" Law.
\end{abstract}

In general terms, one may propose four categories for use when listing species of wild animals. These are : protected fauna, game, aquatic species, and the species listed in agricultural categories. The protected fauna comprises not only those species normally defined as "protected species" (as those listed in art. L.211-1 and L.211-2 of the Rural Code), but also those listed in art. L.212-1 of the same Code (in particular those listed in the Washington Convention Appendixes, and in the European Council ruling $N^{\circ} 3626 / 82$ of 3 December 1982, as amended, for implementation in the European Community). Game includes three categories that fit into each other like Russian dolls : game as in statute-law (the widest category), species for which hunting is allowed, and finally, those liable to be listed as "vermin" by the "Préfets". It should be noted that the last two categories are defined by regulations. Aquatic species lato sensu (including crustaceans and frogs, together with fish and Agnatha) are listed in various documents: freshwaters species subject to fishing laws ; species liable to cause biological imbalance ; species listed in the paragraph 3 of the art. L.23210 of the Rural Code ; non resident species entered in the Ministry's list and whose introduction can be authorised by Prefectorial Decree according to art. R. ${ }^{\star 232-8}$ of the Rural Code ; and other non resident species whose introduction can be authorised by ministerial decision according to art. R. ${ }^{\star} 232-9$ of the Rural Code. Species listed in agricultural categories are also concerned since art. 342 of the Rural Code provides for drawing up a list of "organisms harmful" to plants or plant products, which is different from the list mentioned above which only deals with certain game species.

To be complete, this review also considers some remarkable species categories that are not on these lists, such as: species referred to on negative lists ; certain species omitted in positive lists ; special cases concerning mammals ; some ornamental or aquarium species.

Finally, we examine the possible belonging of one species to various legal categories and the importance of native and introduced species in these categories.

Key-words : legal status, animal species, introduction, aquatic environment, Continental France. 


\section{INTRODUCTION}

Le propos de cet article est double. II consiste à tenter une présentation générale de la structure de classement juridique des espèces de faune sauvage liées aux milieux aquatiques continentaux métropolitains, et à y relever systématiquement le caractère indigène ou non de l'origine biogéographique de ces espèces. En France, en effet, les espèces animales ne sont pas uniquement d'origine indigène et beaucoup sont arrivées plus ou moins tardivement, au cours des derniers siècles en particulier. II s'agit donc de dresser une sorte d' "état des lieux " avant la mise en oeuvre de la loi "Barnier" ( $n^{\circ}$ 95-101 du 2 février 1995), qui a introduit dans le code rural deux articles (L.211-3 et L.211-4) qui traitent des introductions d'espèces dans le milieu naturel. L'hypothèse envisagée est que, dans le dispositif juridique préexistant à la "loi Barnier ", le caractère indigène ou non des espèces et leur répartition au sein des listes juridiques sont liés et s'éclairent mutuellement.

\section{MATÉRIEL ET MÉTHODE}

Au préalable, et afin de clarifier le propos, il y a lieu de signaler, pour les écarter, certaines difficultés. Elles relèvent de deux ordres.

D'abord, la plus ou moins grande ancienneté des introductions - et par conséquent, des éventuelles naturalisations (au sens biogéographique du terme) qui peuvent les avoir suivies occulte une part importante du phénomène en en effaçant le souvenir lorsqu'il se rapporte à certaines espèces présentes depuis longtemps. Cette dimension culturelle, liée au statut d'une espèce dans le "bestiaire imaginaire", est importante : une faible part du public dira spontanément que les carpes ont été introduites par les romains, car la plupart des gens croient vraiment qu'il s'agit d'une espèce indigène. Ensuite, les phénomènes d'introductions d'espèces sont très divers, et il n'est pas toujours aisé de réunir des données pertinentes et suffisantes, ni de distinguer la part qu'y ont prise les hommes de celle qui revient aux organismes considérés ; chez les poissons (ALLARDI et KEITH, 1991 ; KEITH et al., 1992), le cas du silure glane qu'on a longtemps considéré indigène, avant de soutenir le contraire, le montre bien ; chez les mammifères (SAINT GIRONS et al., 1993 ; S.F.E.P.M., 1984), l'exemple du chien viverrin importé d'Extrême-Orient en U.R.S.S. où il a d'abord été naturalisé, puis d'où il a ensuite étendu son aire jusqu'à la France, illustre un autre aspect du problème. II faut aussi avoir conscience du fait que les listes administratives d'espèces ont été rédigées en croisant des données géographiques (présence ou absence sur tel territoire) avec des données de systématique zoologique (oiseaux, insectes, etc...). Y sélectionner les seules espèces écologiquement concernées par ce séminaire implique une part d'arbitraire qui est la conséquence de l'appréciation que l'on porte sur le caractère plus ou moins déterminant de leur liaison aux milieux aquatiques considérés; des cas méritant discussion comme, chez les chauves-souris, ceux des vespertilions de Daubenton et de Capaccini (SAINT GIRONS et al., 1993 ; S.F.E.P.M., 1984), se rencontrent dans des listes appartenant à presque tous les taxons. Enfin, la comparaison entre elles des listes de référence fait apparaître qu'elles ne contiennent pas toutes la même qualité d'information : les deux variables essentielles sont la logique selon laquelle chaque liste a été conçue (pêche ou protection de la nature, par exemple), et le niveau taxonomique pris en compte, car il se situe de la famille à la sous-espèce. Parmi les listes de poissons, par exemple, on rencontre les corégones au niveau du genre, ce qui laisse entière la question du nombre de leurs espèces qui ont été introduites.

En second lieu, il faut souligner que la présentation proposée ici est justifiée de façon précise par les choix retenus pour l'orientation de ce séminaire. Le premier de ces choix porte sur les milieux aquatiques continentaux : une définition des eaux douces, fonctionnelle "sur le terrain ", et qui s'applique à la pleine eau comme aux berges. Une autre limite, de nature biogéographique celle-là, borne nos considérations à la France métropolitaine. La prise en compte des milieux marins ou des territoires et départements d'outre-mer aurait justifié une présentation différente et non de simples ajouts ; il faut donc se garder de généraliser hors du 
cadre considéré. II y a lieu de terminer cet avertissement en signalant le piège des synonymies: le terme "introduction " figure dans le livre II (nouveau) du code rural avec au moins trois sens distincts : à côté des articles L.211-3 et L.211-4 déjà cités, qui connotent l'arrivée d'une espèce nouvelle dans une aire biogéographique ou un milieu donné, l'article L.212-1 emploie " introduction " au sens douanier du terme (qui est à rapprocher de l'idée d'importation) ; quant à l'article L.232-10, il l'utilise au sens de mise ou de remise à l'eau dans une catégorie juridique de milieux précise (les eaux soumises à tels ou tels articles du code rural).

Sous les réserves exposées ci-dessus, on peut proposer de se référer à quatre grandes catégories de listes d'espèces pour le classement juridique de la faune sauvage : les espèces de faune protégée, les gibiers, les animaux de pêche, et enfin les espèces relevant de classifications agricoles.

Dans chacune de ces catégories, on a recensé les principales listes contenant des espèces liées aux milieux aquatiques continentaux métropolitains et, au sein de chaque liste, on a noté ces espèces. La base ainsi constituée comporte 24 unités de référence : 2 textes internationaux (la convention de Washington et le règlement européen pris pour son application), un ensemble jurisprudentiel (le gibier), un texte législatif (lié à la pêche), et 20 textes réglementaires de droit interne. Elle a permis d'examiner le lien entre le statut juridique des espèces et le caractère indigène ou non de leur origine biogéographique.

\section{LA FAUNE PROTÉGÉE}

La faune protégée ne comprend pas seulement les animaux d'espèces auxquelles on réserve le plus souvent, quoiqu'abusivement, l'expression d'“ espèces protégées ", à laquelle s'attache juridiquement l'outil technique de l'interdiction. Elle comprend aussi des espèces que l'on protège en soumettant à autorisation les activités susceptibles de leur porter atteinte.

\subsection{Les mesures d'interdiction}

Au sens restreint mais habituel du terme, les "espèces protégées " sont celles dont la conservation est justifiée par un intérêt scientifique particulier ou par les nécessités de la préservation du patrimoine biologique. Elles figurent sur des listes prises au titre des articles L.211-1 et L.211-2 du code rural (numéros donnés par la codification d'avril 1991 aux articles 3 et 4 de la loi $n^{\circ} 76-629$ du 10 juillet 1976 relative à la protection de la nature). Ces deux articles permettent d'interdire la destruction ou l'enlèvement des oeufs ou des nids, la mutilation, la destruction, la capture ou l'enlèvement, la perturbation intentionnelle, la naturalisation d'animaux de ces espèces ou, qu'ils soient vivants ou morts, leur transport, leur colportage, leur utilisation, leur détention, leur mise en vente, leur vente ou leur achat. Une telle énumération montre bien que c'est prioritairement sur le terrain, en s'attachant à la protection des animaux qui y vivent "en liberté ", que cet article vise à assurer la protection des espèces européennes. Elles appartiennent à des taxons zoologiques assez divers : Mollusques, Arthropodes (crustacés et insectes), Vertébrés surtout (agnathes, poissons, amphibiens, reptiles, oiseaux et mammifères) et elles figurent dans des arrêtés du ministre chargé de la protection de la nature qui précisent les interdictions particulières se rapportant à chacune d'elles, selon, le cas échéant, l'article dont elles relèvent. Quoique dans leur immense majorité il s'agisse bien d'espèces indigènes, on y trouve également certaines espèces qui pourraient avoir été introduites, comme l'émyde lépreuse parmi les reptiles (S.H.F., 1989), le discoglosse peint parmi les amphibiens (idem), ou l'ide mélanote parmi les poissons (ALLARDI et KEITH, 1991 ; KEITH et al., 1992), et aussi des animaux tels que le vison d'Europe, espèce pourtant emblématique, dont l'atlas des mammifères sauvages de France (S.F.E.P.M., 1984) indique qu'aucune mention antérieure à 1839 ne serait connue en France et que la première mention espagnole daterait de 1951. Une extension aux départements d'outre-mer du champ d'intérêts de ce séminaire aurait permis d'y mettre en évidence (avec les tortues guadeloupéennes, par exemple) la confirmation de ces observations. 


\subsection{Les activités soumises à autorisation}

D'autres listes d'espèces protégées sont prises en application de l'article L.212-1 du code rural (qui correspond à l'ancien article 5 de la loi relative à la protection de la nature). Cet article permet de soumettre à autorisation la production, la détention, la cession à titre gratuit ou onéreux, l'utilisation, le transport, l'introduction quelle qu'en soit l'origine, l'importation sous tous régimes douaniers, l'exportation et la réexportation de tout ou partie d'animaux d'espèces non domestiques et de leurs produits. Etant donné qu'il s'agit de réglementer, c'est-à-dire de préciser les conditions d'exercice et les limites d'activités qui ne sont pas interdites, on trouve ces listes d'espèces dans des textes d'interface entre la protection de la nature et le commerce international ou encore l'importation ou la commercialisation de gibiers ou d'animaux de pêche. Ces espèces relèvent également de taxons assez variés : Annélides, Arthropodes (crustacés), Vertébrés (poissons, amphibiens, oiseaux et mammifères), pour s'en tenir aux seules espèces européennes. En effet, et contrairement aux espèces protégées en vertu des articles L.211-1 et L.211-2, il n'y a pas de lien logique qui doive nécessairement lier au patrimoine naturel des pays de l'Europe (en pratique, par l'occupation permanente ou régulière de leur territoire), les espèces que protège l'article L.212-1. Ainsi, c'est en vertu de cet article L.212-1 du code rural qu'un arrêté du 1er mars 1993 étend les dispositions pénales prévues par ce code pour la protection de la faune aux espèces citées en annexe à la convention de Washington sur le commerce international des espèces menacées d'extinction et au règlement européen pris pour son application. On peut noter que les espèces de la faune européenne citées dans ces deux textes sont souvent migratrices, mais pas introduites.

\subsection{Domaine de recouvrement de ces deux types de protection}

Qu'elles soient prises en application des articles L.211-1 et L.211-2 ou en vertu de l'article L.212-1, ces listes ne sont pas exclusives les unes des autres. Le cas de l'espèce indigène d'esturgeon, Acipenser sturio, illustre bien l'usage qui est fait des possibilités complémentaires qu'offrent ces deux sources.

Au titre des articles L.211-1 et L.211-2, un arrêté du 25 janvier 1982 interdit sur tout le territoire national la destruction ou l'enlèvement des oeufs, la destruction, la mutilation, la capture, l'enlèvement, la naturalisation ou le transport, l'utilisation, la mise en vente, la vente ou l'achat des animaux de cette espèce provenant du territoire national ; il réserve la capture, le prélèvement, le transport, la vente ou l'achat aux seules opérations spécialement autorisées à des fins scientifiques ou de repeuplement. Ce premier arrêté agit donc par le moyen de la protection des spécimens. Toujours au titre des mêmes articles du code rural, un arrêté du 8 décembre 1988 complète ce dispositif en faisant figurer cette espèce dans une liste de poissons dont il est interdit de détruire, d'altérer ou de dégrader les milieux particuliers et notamment les lieux de reproduction désignés par arrêté préfectoral. Ce second arrêté agit donc au moyen de la protection des biotopes.

Or, parallèlement et de façon complémentaire, l'activité du commerce international est réduite aux seules exceptions justifiées par certaines fins scientifiques ou de repeuplement, grâce au classement de cette espèce (en annexe I) par la convention de Washington dont les pénalités sont mises en œuvre au titre de l'article L.212-1.

\section{LE GIBIER}

\subsection{Un ensemble défini par la jurisprudence}

Aucun texte ne donne la définition du gibier : c'est la jurisprudence qui l'établit. En pratique, le gibier au sens jurisprudentiel du terme comprend toutes les espèces d'oiseaux et de mammifères de France métropolitaine qui y sont ou y ont été chassées. Elles comprennent donc, notamment, la plupart des espèces de mammifères de taille égale ou supérieure à celle d'un écureuil, ainsi que toutes les espèces indigènes d'oiseaux. D'un point de vue administratif, elles sont réparties en trois ensembles qui s'emboîtent comme des poupées russes : les gibiers 
au sens de la jurisprudence (le plus vaste), les espèces dont la chasse est autorisée, et enfin celles d'entre elles qui sont susceptibles d'être déclarées nuisibles par les préfets. Le décalage entre ces deux derniers ensembles, définis par la voie réglementaire, et la définition générale du gibier, donnée par la jurisprudence, explique ce qui semble être un paradoxe à souligner : un grand nombre des espèces de gibier ne sont pas soumises actuellement à la chasse. C'est le cas, en particulier, de ceux des gibiers qui figurent sur les listes d'" espèces protégées ", comme le castor, la loutre ou le martin-pêcheur.

\subsection{Les espèces de gibier que l'on peut chasser}

Par un arrêté en date du 26 juin 1987, le ministre chargé de la chasse a fixé la liste limitative des espèces de gibier dont la chasse est autorisée. Sous les réserves liées aux limites du champ que détermine l'orientation du présent séminaire (espèces animales liées aux milieux aquatiques continentaux métropolitains), on note que dans cette liste, les espèces d'oiseaux et celles de mammifères diffèrent par leurs origines: alors que les oiseaux sont indigènes, les mammifères, à l'exception du putois, sont introduits. Si certains d'entre eux (comme le vison d'Amérique, dont des spécimens échappés d'élevages se seraient acclimatés dès le début du siècle) sont actuellement représentés par des populations importantes, d'autres (comme le raton laveur) sont loin d'avoir connu cette expansion (SAINT GIRONS et al., 1993 ; S.F.E.P.M., 1984).

\subsection{Les espèces susceptibles d'être classées nuisibles}

Au titre de l'article L.227-8 du code rural, les espèces dont il est question ici sont des animaux contre lesquels les particuliers peuvent être autorisés à mener des opérations de destruction. L'arrêté du 30 septembre 1988 fixe la liste des animaux (gibiers) susceptibles d'être déclarés nuisibles. On peut remarquer que le nombre de ces espèces d'oiseaux nuisibles est beaucoup plus faible que celui des espèces d'oiseaux que l'on peut chasser (il est même nul ou, au plus, insignifiant, dans le cadre des espèces de milieux aquatiques continentaux métropolitains), alors que la liste des mammifères liés aux eaux continentales métropolitaines est identique dans les deux arrêtés. En tout état de cause, l'opposition dans l'origine biogéographique entre oiseaux et mammifères de gibiers dont la chasse est autorisée se retrouve dans la liste des espèces susceptibles d'être déclarées nuisibles. C'est dans cette liste limitative que, chaque année, le préfet de chaque département retient celles des espèces qu'il classe nuisibles par arrêté.

\section{LES ANIMAUX DE PÊCHE}

Les animaux de pêche, au sens large (qui regroupent certains crustacés et des grenouilles avec les poissons et les agnathes), font l'objet de listes diverses : espèces représentées dans les eaux douces soumises à la législation sur la pêche ; espèces susceptibles de provoquer des déséquilibres biologiques; espèces citées au $3^{\circ}$ de l'article L.232-10 du code rural; espèces non représentées, mais figurant sur la liste ministérielle de celles dont l'introduction peut être autorisée en vertu d'un arrêté préfectoral pris au titre de l'article R. * 232-8 du code rural ; autres espèces non représentées, mais d'introduction autorisée en vertu d'une décision ministérielle prise au titre de l'article R. ${ }^{*} 232-9$ du code rural.

\section{la pêche}

3.1. Les espèces représentées dans les eaux douces soumises à la législation sur

Les eaux douces soumises à tout ou partie de la législation sur la pêche comprennent les eaux libres, c'est-à-dire tous les cours d'eau, canaux, ruisseaux, ainsi que les plans d'eau avec lesquels ils communiquent (y compris les piscicultures et les enclos piscicoles) et, par application de l'article R.231-5 du code rural, certaines eaux closes soumises à cette législation à la demande de leurs propriétaires. La liste des espèces qui y sont représentées est fixée par un arrêté du 17 décembre 1985 : elles ne comprennent que quelques Crustacés et des Vertébrés (agnathes, poissons et amphibiens). II s'agit actuellement d'une liste nationale unique 
et non de listes établies par bassin, ce qui renverrait à une notion différente du concept d'introduction. Dressée en application du $2^{\circ}$ de l'article L.231-10, cette liste a pour fonction d'interdire, dans les eaux soumises à la législation sur la pêche, l'introduction des espèces qui n'y sont pas énumérées. Plus du quart de ces espèces (comme la carpe ou le sandre) ne sont pas indigènes.

\subsection{Les espèces susceptibles de provoquer des déséquilibres biologiques}

Etablie à l'article R. ${ }^{*} 232-3$ du code rural en application du $1^{\circ}$ de l'article L.232-10, cette liste de poissons, d'amphibiens et de crustacés est constituée de façon originale : les crustacés et les poissons font l'objet d'une liste positive, qui comprend trois espèces seulement : le crabe chinois, le poisson-chat et la perche soleil. En revanche, les grenouilles et les écrevisses sont traitées négativement, en référence à des listes limitatives dont elles sont exclues par la formule : "les espèces autres que... ". Dans cette liste d'espèces susceptibles de provoquer des déséquilibres biologiques, aucune n'est indigène. II est intéressant de noter que le décret du 16 septembre 1958, abrogé, dont elle est issue utilisait la formule " particulièrement nuisibles " et que sa liste comprenait en outre le hotu et, pour les eaux de première catégorie (c'est-à-dire les eaux à truites), l'anguille, poisson indigène.

\subsection{Les espèces citées au $3^{\circ}$ de l'article L.232-10 du code rural}

Les espèces citées au $3^{\circ}$ de l'article L.232-10 du code rural comprennent cinq poissons figurant sur la liste des espèces représentées dans les eaux douces nationales ; tous sont carnassiers et il est interdit de les introduire dans la plupart des eaux classées en première catégorie. II s'agit du brochet et de la perche (indigènes), mais aussi du sandre et des deux black-bass (introduits). Les espèces introduites sont donc en majorité dans cette liste.

3.4. Les espèces non représentées, mais figurant sur la liste ministérielle de celles dont l'introduction peut être autorisée en vertu d'un arrêté préfectoral pris au titre de l'article R. ${ }^{\star 2} 232-8$ du code rural

Par définition, il s'agit d'espèces introduites ; c'est effectivement le cas puisque les deux espèces concernées sont le saumon coho du Pacifique et l'esturgeon de Sibérie.

3.5. Les autres espèces non représentées, mais d'introduction autorisée en vertu d'une décision ministérielle prise au titre de l'article R.`232-9 du code rural

Cette disposition a été expressément prévue et réservée pour des cas de recherches scientifiques. Comme la précédente, et par définition également, elle paraît ne devoir être appliquée qu'à des espèces introduites. Néanmoins, ce cas de figure juridique ne semble pas avoir déjà été utilisé et il n'existe donc pas d'exemple sur lequel s'appuyer.

\section{LES ORGANISMES NUISIBLES AUX VÉGÉTAUX OU AUX PRODUITS VÉGÉTAUX}

Les réglementations agricoles interviennent aussi, dans la mesure où l'article 342 du code rural prévoit que le ministre de l'agriculture dresse la liste des organismes nuisibles contre lesquels la lutte est organisée dans les conditions qu'il fixe. Cette liste diffère de celle - déjà évoquée - que dresse le ministre chargé de la chasse et qui concerne exclusivement des gibiers. Le champ taxonomique des dispositions qui peuvent être prises au titre de la protection des végétaux est théoriquement très vaste, puisque l'article 342 du code rural prévoit que "sont considérés comme des organismes nuisibles tous les ennemis des végétaux ou des produits végétaux, qu'ils appartiennent au règne animal ou végétal ou se présentent sous forme de virus, mycoplasmes ou autres agents pathogènes $"$.

En pratique toutefois, les réglementations agricoles ne paraîssent influencer le statut que de bien peu d'espèces susceptibles d'entrer dans le champ de ce séminaire. On peut citer le rat musqué (dont un arrêté du 15 décembre 1951 interdit l'importation, l'élevage et le transport à l'état vivant) et peut-être, car de façon indirecte (puisque c'est par le détour de textes réglementant leur empoisonnement, au titre de la loi $n^{\circ} 525$ du 2 novembre 1943, validée et 
modifiée, sur l'homologation des produits de défense contre les ennemis des cultures), le rat surmulot, le ragondin et le campagnol terrestre. Un arrêté du 12 juillet 1979 réglemente l'empoisonnement du ragondin et du campagnol terrestre. Par ailleurs, l'empoisonnement du rat surmulot fait l'objet de l'arrêté du 26 avril 1988, modifié par un arrêté du 21 août 1991 ; cet arrêté modificatif est le seul de ces textes dont le ministre de l'environnement est cosignataire.

Le rat surmulot serait arrivé de Chine entre le XVlème et le XVIllème siècle (SAINT GIRONS et al., 1993 ; S.F.E.P.M., 1984) et on a signalé plus haut que le rat musqué et le ragondin ont été introduits ; de ces quatre espèces, seul le campagnol terrestre est donc réputé indigène.

\section{LES ESPÈCES NE FIGURANT PAS DANS LES LISTES POSITIVES CI-DESSUS}

Par défaut, et à titre complémentaire, on peut aussi repérer et examiner diverses catégories remarquables d'espèces non listées ci-dessus.

\subsection{Les espèces non mentionnées, qu'un texte vise pourtant a contrario :}

5.1.1. C'est le cas des espèces d'écrevisses et de grenouilles visées par l'article R. ${ }^{* 232-}$ 3 du code rural pour être susceptibles de provoquer des déséquilibres biologiques dans certaines eaux où leur introduction est interdite. On peut citer notamment la grenouille américaine Rana catesbeiana et au moins trois espèces d'écrevisses américaines (VIGNEUX et al., 1993) ;

5.1.2. ainsi, également, les espèces de pêche non citées dans la liste de celles qui sont représentées sur le territoire national, mais qui sont pourtant connues pour y être présentes et s'y reproduire spontanément (comme le chabot de Petit, réputé indigène, ou le Pseudorasbora parva, originaire du Japon) ;

5.1.3. de même, les espèces de pêche non citées dans la liste de celles qui sont représentées sur le territoire national, mais connues pour y être néanmoins présentes en quantités importantes, bien qu'elles ne s'y reproduisent pas spontanément (comme les " carpes chinoises"). A noter, en matière d'écrevisses et de grenouilles, la redondance entre les espèces visées ci-dessus au paragraphe 5.1.1. et celles visées aux paragraphes 5.1.2. ou 5.1.3.

5.2. Des espèces qui, sans être explicitement visées, ne figurent pas sur certaines listes positives

C'est le cas du bruant des roseaux, oiseau indigène donc gibier au sens de la jurisprudence, mais ne figurant ni sur les listes de gibier que l'on peut chasser, ni sur celles des espèces protégées.

\subsection{Certains cas particuliers d'espèces de mammifères}

On peut citer le rat des moissons, hôte des roselières notamment : d'origine asiatique, il serait néanmoins présent depuis le pléistocène, selon SAINT GIRONS (in S.F.E.P.M., 1984). De telles espèces ne semblent pas avoir de statut, au sens propre du terme.

\subsection{Certains animaux d'ornement ou d'aquariophilie}

L'exemple le plus généralement cité aujourd'hui est celui de la tortue de Floride, ce qui permet d'ailleurs un parallèle intéressant avec le poisson rouge ou carassin doré : voilà deux espèces importées dont les spécimens s'acclimatent facilement. Arrivé à partir du XVIlème siècle, le carassin doré s'est naturalisé et figure désormais, comme plus de vingt autres espèces non indigènes (ALLARDI et KEITH, 1991 ; KEITH et al., 1992), sur la liste des espèces représentées dans les eaux douces nationales. On ignore encore, en revanche, si la tortue de Floride, d'importation récente, constituera des populations reproductives viables, condition biologiquement nécessaire à son éventuelle naturalisation. 
Bien que les espèces que l'on vient d'évoquer aient pour caractéristique commune de ne pas figurer sur les listes mentionnées dans cet article, toutes ne sont pourtant pas dépourvues de statut juridique. Ainsi, le bruant des roseaux peut être considéré comme une espèce objectivement protégée, puisqu'il bénéficie d'un statut de gibier renforcé par le fait que sa chasse n'est pas ouverte. De façon analogue d'un point de vue juridique, même si cette autre classification aboutit à un résultat opposé du point de vue de la gestion de l'espèce, ni Rana catesbeiana, ni les trois écrevisses américaines évoquées à l'occasion des espèces susceptibles de provoquer des déséquilibres biologiques ne peuvent être introduites dans les eaux douces soumises à la législation sur la pêche; elles sont donc, elles aussi, soumises à un régime juridique précis. Cette remarque vaut aussi bien pour toutes les espèces de poissons, de crustacés et de grenouilles qui ne figurent pas sur la liste des espèces représentées dans les eaux douces nationales, et qui y sont pourtant présentes.

\section{CONCLUSION}

Dans les limites de l'examen des seules espèces animales liées aux milieux aquatiques continentaux métropolitains, et pour sommaire qu'il soit, ce tour d'horizon d'une réglementation complexe permet la mise en évidence de deux points remarquables.

D'abord, certaines catégories d'espèces sont incluses dans d'autres catégories plus vastes, selon une hiérarchie stricte (ainsi, le putois et le vison d'Amérique, par exemple, figurent sur la liste des animaux susceptibles d'être classés nuisibles, qui eux-mêmes font actuellement partie des gibiers que l'on peut chasser, qui à leur tour font partie de la catégorie encore plus vaste des gibiers au sens de la jurisprudence). Certaines autres catégories sont susceptibles de recouvrement mutuel. C'est le cas, l'un par rapport à l'autre, des deux sous-ensembles qui constituent la faune protégée (sous-ensembles caractérisés, l'un par l'interdiction, l'autre par la soumission à autorisation de certaines activités relatives à ces espèces) ; ainsi, l'esturgeon commun figure non seulement sur un arrêté pris au titre des articles L.211-1 et L.211-2 du code rural, mais aussi en annexe I de la convention de Washington dont la mise en oeuvre bénéficie des dispositions pénales prévues par le code rural pour la protection de la faune en vertu d'un arrêté du 1er mars 1993, pris au titre de l'article L.212-1 de ce code. C'est aussi le cas entre grandes catégories, par exemple entre faune protégée et animaux de pêche (comme pour l'esturgeon, encore une fois) ou encore, entre faune protégée et gibier (comme le montrent les exemples du castor ou du martin-pêcheur).

Deuxièmement, on observe des catégories à vocation générale (telles que le gibier au sens jurisprudentiel ou bien les animaux mentionnés sur la liste des espèces de pêche représentées dans les eaux douces), qui forment des ensembles réunissant un certain nombre d'espèces, indépendamment de leur origine biogéographique. A l'inverse, on note une corrélation entre ce caractère et la répartition des espèces animales au sein de certaines autres listes. Ainsi, les espèces indigènes ont le quasi-monopole des listes d' "espèces protégées " (même si, contrairement à une fausse évidence intuitive, ces listes ne leur sont pas strictement réservées), alors qu'en revanche, les espèces introduites sont majoritaires dans les diverses catégories se référant avec plus ou moins de précautions au concept de nuisibilité : gibiers "nuisibles", animaux de pêche "susceptibles de provoquer des déséquilibres biologiques" (ex-« particulièrement nuisibles ") et espèces citées au $3^{\circ}$ de l'article L.232-10 du code rural, " organismes nuisibles " du point de vue de la protection des végétaux.

Cette dernière observation appelle deux remarques. D'abord, il y a lieu de rappeler que la nuisibilité ne peut se penser indépendamment de l'identification claire (donc de la justification argumentée) des intérêts par l'atteinte desquels on entend la définir, ainsi que des critères qui permettent de l'identifier et de la mesurer. Ce point est essentiel : une telle exigence de rigueur vaut pour la composition de toute liste d'espèces. Ensuite, l'inscription d'une espèce introduite sur l'une de ces listes à caractère nuisible plus ou moins souligné peut être interprétée à la fois comme la marque de son rejet, mais aussi comme la reconnaissance juridique de sa naturalisation biogéographique (qu'elle soit déjà effective ou encore potentielle). Une analyse 
historique du statut culturel et juridique des espèces de faune sauvage permettrait peut-être d'apprécier dans quelles conditions ce type de régimes se révèle être aussi une étape, une sorte de premier pas, vers un statut juridique ultérieur de reconnaissance de légitimité, comme dans le cas du hotu, précédemment cité pour n'avoir pas été intégré à la liste des espèces de poissons susceptibles de provoquer des déséquilibres biologiques, alors qu'il figurait pourtant dans l'" ancêtre " de cette liste : celle des "espèces particulièrement nuisibles". On voit par là que les questions liées à l'évolution des statuts et des listes d'espèces méritent une attention particulière.

\section{REMERCIEMENTS}

Que tous les amis et collègues qui, par leurs discussions, m'ont aidé à l'élaboration de ce texte, trouvent ici l'expression de ma reconnaissance. Je remercie en particulier Madame Marie-Angèle HERMITTE ainsi que Messieurs Jean ALLARDI, Gérard GROLLEAU et Cyrille de KLEMM pour leur lecture de ce manuscrit et les remarques judicieuses qu'ils m'ont faites.

\section{BIBLIOGRAPHIE}

ALLARDI J., KEITH P., 1991. Atlas préliminaire des poissons d'eau douce de France. Coll. Patrimoines Naturels, vol. 4, M.N.H.N. / S.F.F., CEMAGREF, Min. Env., Paris, 232 p.

KEITH P., ALLARDI J., MOUTOU B., 1992. Livre rouge des espèces menacées de poissons d'eau douce de France métropolitaine et bilan des introductions. M.N.H.N. / S.F.F., CEMAGREF, C.S.P., Min. Env., Paris, 120 p.

SAINT GIRONS M.C., MAURIN H., ROSOUX R., KEITH P., 1993. Les mammifères d'eau douce ; leur vie, leurs relations avec l'homme. Ministère de l'Environnement, Ministère de l'Agriculture et de la Pêche et S.F.E.P.M., Paris, 48 p.

S.F.E.P.M. [FAYARD A., dir.], 1984. Atlas des Mammifères sauvages de France. Min. Env. / D.P.N., M.N.H.N. / S.F.F., Paris, 299 p.

S.H.F. [CASTANET J., GUYETANT R., coord.], 1989. Atlas de répartition des amphibiens et reptiles de France. Secrétariat d'Etat Env. / D.N.P., M.N.H.N. / S.F.F., Paris, 191 p.

VIGNEUX E., KEITH P., NOEL P., 1993. Atlas préliminaire des Crustacés Décapodes d'eau douce de France. Coll. Patrimoines Naturels, Vol. 14, S.F.F., B.I.M.M.-M.N.H.N., C.S.P., Min. Env., Paris, $55 \mathrm{p}$.

\section{RÉFÉRENCES BIBLIOGRAPHIQUES COMPLÉMENTAIRES}

La plupart des textes juridiques à consulter sont réunis dans les recueils usuels désignés ci-après (relatifs à des textes évolutifs du droit positif, ces usuels sont, par définition, incomplets ; les dates de dernière édition ne sont donc ici indiquées que pour signaler a contrario le caractère caduc des précédentes éditions) :

Code rural code forestier, mai 1995, Dalloz.

Protection de la nature. Journal officiel de la République française. Brochure $n^{\circ} 1454$ :

Tome I: Protection de la faune et de la flore, 608 p., novembre 1994.

Tome III : Pêche en eau douce et gestion des ressources piscicoles, 583 p., mars 1991.

Tome V : Convention de Washington - Textes communautaires et internationaux, 415 p., septembre 1990 .

\section{Brochure $\mathrm{n}^{\circ} 1681$ : La chasse, 561 p., juin 1996.}

Nota bene : une édition à jour de la convention de Washington est disponible au Ministère de l'Environnement / Direction de la Nature et des Paysages; la dernière est datée du 15 mars 1995. 